\title{
A two-cilia model for vertebrate left-right axis specification
}

\author{
Clifford J. Tabin ${ }^{1}$ and Kyle J. Vogan ${ }^{2}$ \\ Department of Genetics, Harvard Medical School, Boston, Massachusetts 02115, USA
}

The mechanisms underlying vertebrate left-right (L-R) axis specification have attracted much interest among developmental biologists (for review, see Burdine and Schier 2000; Capdevila et al. 2000). Of particular interest from a conceptual standpoint is the question of how L-R positional information first originates within the context of a bilaterally symmetric embryo. To achieve the consistent L-R handedness characteristic of the vertebrate body plan, the L-R axis must be reliably oriented with respect to the other two embryonic axes, anteroposterior (A-P) and dorsoventral (D-V; Brown and Wolpert 1990). Understanding how embryos achieve this goal without reference to any external cues represents a formidable challenge for embryologists and theoretical biologists alike.

The first breakthrough in understanding the molecular basis of handed asymmetry was the discovery of a cascade of signals produced asymmetrically in the early embryo that was sufficient to direct the orientation of later L-R morphogenetic events (Levin et al. 1995). This, together with subsequent reports identifying additional genes expressed asymmetrically in the early embryo, opened the door to ongoing work directed at understanding how L-R positional information is propagated in the embryo as well as how this information is ultimately translated into morphological manifestations of L-R asymmetry (for review, see Burdine and Schier 2000; Capdevila et al. 2000). Perhaps most important, however, were the general patterns of asymmetric gene expression first reported by Levin et al. (1995)—initial tightly localized domains of asymmetric gene expression at the chick node (ActRIIa, Shh, and then Nodal medially) followed by broad domains of asymmetric gene expression throughout the lateral plate (Nodal in the left lateral plate mesoderm). Significantly, although some of the specific molecular players appear to vary across different species, the node has consistently been the location where the earliest molecular asymmetries are centered, drawing attention to this region as the most likely site for the initial symmetry breaking event responsible for specifying the orientation of the L-R axis /Vogan and

Corresponding authors.

${ }^{1}$ E-MAIL tabin@rascal.med.harvard.edu; FAX (617) 432-7595.

2vogan@rascal.med.harvard.edu; FAX (617) 432-7595.

Article and publication are at http://www.genesdev.org/cgi/doi/10.1101/ gad.1053803.
Tabin 1999; Capdevila et al. 2000). The precise manner in which asymmetric gene expression might first be established, however, remained a mystery until a second breakthrough discovery documenting the existence of motile monocilia and directional fluid flow at the mouse node (Nonaka et al. 1998).

\section{Some node cilia are motile ...}

There has long been a link between ciliary motility and the regulation of L-R asymmetry. In particular, humans with Kartaganer syndrome exhibit frequent mirror-image reversals in the orientation of their internal organs along with respiratory difficulties caused by immotile tracheal cilia and male infertility related to defects in sperm motility (Afzelius 1976). This syndrome has recently been shown to result from any of a series of mutations in components of the ciliary motor, including dynein intermediate chains (Pennarun et al. 1999; Guichard et al. 2001) and dynein heavy chains (Bartoloni et al. 2002; Olbrich et al. 2002). This association between primary ciliary dyskinesia and the regulation of L-R asymmetry was strengthened further by the discovery that the inversus viscerum (iv) mouse, which exhibits frequent L-R reversals, results from a mutation in a ciliary dynein heavy chain gene $(\operatorname{Lrd})$ expressed specifically in node cells (Supp et al. 1997). Notably, the existence of monocilia at the mouse node had been discovered several years earlier (Sulik et al. 1994; Bellomo et al. 1996). Researchers in the field, however, did not immediately connect the existence of these node cilia to the initiation of L-R asymmetry, perhaps because of conflicting reports in the literature surrounding the motile properties of these cilia (Sulik et al. 1994; Bellomo et al. 1996). In this respect, the paper by Hirokawa and colleagues (Nonaka et al. 1998) first describing a cilia-generated leftward flow at the mouse node sharply refocused thinking in the field, as prior to this time few actually believed that a mechanical mechanism involving directional fluid flow might lie at the heart of vertebrate L-R axis specification. Nonetheless, this realization was quite satisfying on an intellectual level as it provided a framework for understanding how handedness might arise de novo from the fixed orientation of the cilia coupled with the molecular chiralities acting to constrain the direction of ciliary rotation (Vogan and Tabin 1999).

The key data reported by Hirokawa and colleagues 
(Nonaka et al. 1998) — that node cilia are motile, that directional fluid flow exists at the mouse node, and that laterality defects in mice lacking the ciliary assembly protein KIF3B coincide with a lack of node cilia and an absence of nodal flow-indicated an important role for nodal flow in the specification of the vertebrate L-R axis. In turn, one straightforward prediction of the nodal flow model was that mice deficient in Lrd function should show defects in ciliary motility and in the generation of nodal flow. This was an important prediction to test because mice lacking KIF3B (Nonaka et al. 1998) or the related ciliary assembly protein KIF3A (Marszalek et al. 1999; Takeda et al. 1999) are essentially devoid of cilia, making it unclear whether it is the absence of ciliary motility (and the concomitant loss of nodal flow) or the lack of cilia per se that is responsible for the L-R patterning defects observed in these mice. It was demonstrated shortly afterward that embryos deficient in Lrd function, although exhibiting normal node morphology, contain immotile node cilia and lack any discernable flow in the node region, lending key support to the nodal flow model (Okada et al. 1999; Supp et al. 1999). In a technically challenging converse experiment, it was also shown that directional flow, when supplied exogenously to the nodes of iv mice, is sufficient to specify the orientation of the L-R axis in otherwise randomized embryos (Nonaka et al. 2002). Strong rightward flow is sufficient, moreover, to reverse L-R axis orientation in wild-type mouse embryos (Nonaka et al. 2002). Collectively, these studies demonstrate that directional fluid flow is both necessary and sufficient for specifying the L-R axis in the mouse, lending strong experimental support to the nodal flow model of L-R axis specification.

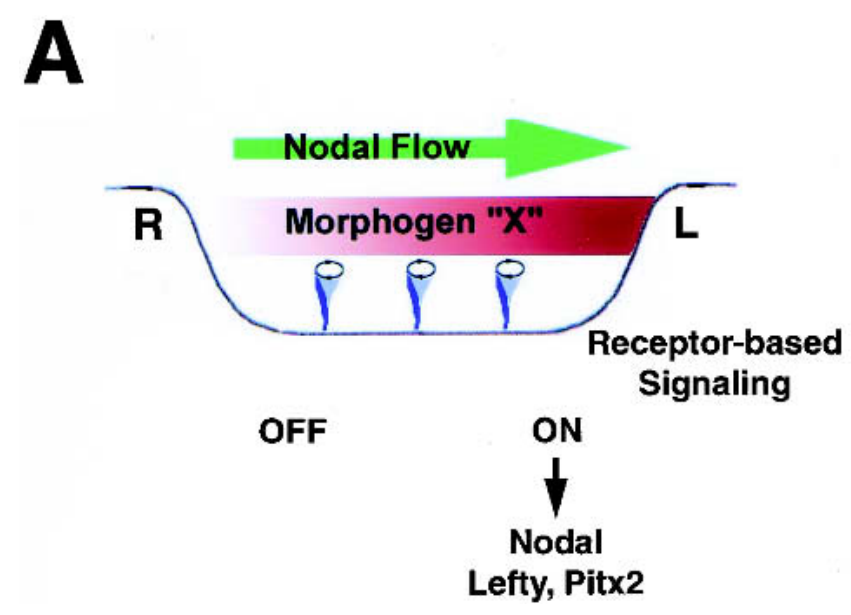

These experiments left unanswered, however, the question of how directional fluid flow at the node might trigger differential cascades of gene expression on the left and right sides of the embryo. One solution to this problem, put forward by Hirokawa and colleagues (Nonaka et al. 1998; Okada et al. 1999|, suggests that the observed leftward flow is directly responsible for the asymmetric redistribution of one or more symmetrically released signaling molecules in the vicinity of the node, resulting in the differential accumulation of these solubilized factors to the left of the node and the initiation of distinct downstream signaling events on the left side of the embryo. This asymmetric redistribution of key signaling molecules-the so-called "morphogen flow" hypothesis (Fig. 1A) - has been the prevailing view held to date by most proponents of the nodal flow model. Furthermore, several secreted factors produced in the node region, including Fgf8 (Meyers and Martin 1999), Gdf1 (Rankin et al. 2000), and Nodal (Brennan et al. 2002), have been proposed to function as this critical node morphogen, primarily based on the observed loss of left-specific gene expression in mice carrying targeted deletions of these genes. It is important to point out, however, that no empirical evidence exists for the differential distribution of these proteins in the node region in response to nodal flow, and no other experimental validation for this model has been put forward to date.

\section{... But are motile cilia enough?}

Whereas at first glance the morphogen flow hypothesis might appear attractive on the basis of its inherent simplicity, a more careful consideration of the hypothesis

\section{B}

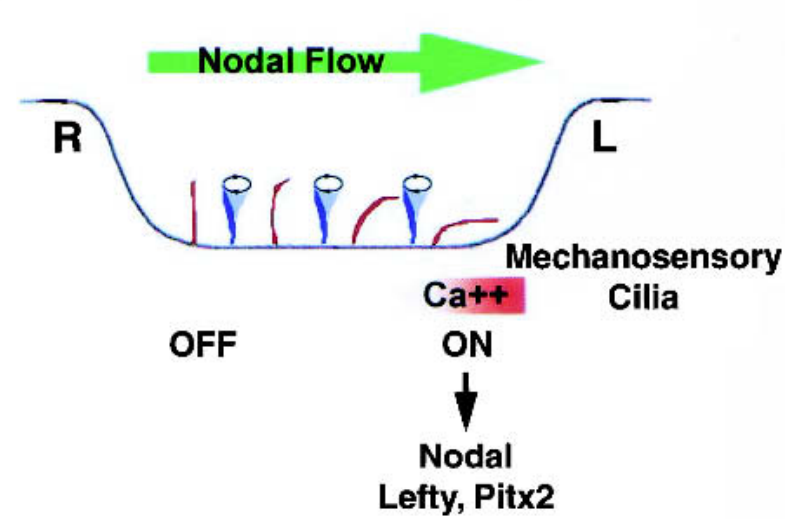

Figure 1. Alternative models of the proposed signaling events functioning downstream of nodal flow. $(A)$ According to the morphogen flow hypothesis (Nonaka et al. 1998; Okada et al. 1999), a critical left determinant, morphogen "X" (red), is released symmetrically into the node region and subsequently becomes concentrated to the left side of the node in response to nodal flow (green arrow) generated by the clockwise vortical rotation of motile node cilia (blue). Morphogen "X" subsequently interacts with its receptor on the left side of the node to initiate expression of left-sided genes such Nodal, Lefty, and Pitx2. (B) According to the two-cilia model described here, nodal flow (green arrow) generated by the activity of motile node cilia (blue) produces differential fluid pressure at the two sides of the node, leading to asymmetric stimulation of mechanosensory cilia (red) distributed symmetrically in the node region. In response to stimulation, these mechanosensory cilia initiate a calcium-mediated signal transduction event that leads to the asymmetric induction of Nodal and other left-sided genes. Both panels illustrate transverse sections through the node region (ventral to the top). L, left; R, right. 
reveals some serious problems that are difficult to reconcile with existing data, suggesting that the morphogen flow hypothesis, at least in its simplest form, cannot be correct. The first issue concerns the plausibility of redistributing symmetrically expressed factors in response to bulk fluid flow. Although there are several ways to consider this issue from a fluid dynamic standpoint, perhaps the strongest objection can be raised in light of recent experiments in which flow was artificially directed across the surface of the node in culture (Nonaka et al. 2002). Although this study definitively demonstrated that the direction of fluid movement across the node is sufficient to determine the orientation of the L-R axis (as discussed above), it is troubling that varying the absolute flow rate across the node (i.e. slow leftward vs. fast leftward flow) did not alter situs (Nonaka et al. 2002). In particular, if a critical L-R morphogen (i.e., one that could indeed be redistributed across the node in response to fluid flow) were in fact being released at the node, one would expect that a sufficient flux of fluid would simply wash it away, resulting in L-R randomization rather than normal situs.

A second objection to the morphogen flow hypothesis stems from the observed differences in the molecular phenotypes of mice harboring immotile node cilia versus those devoid of node cilia altogether. In the case of the former (i.e., iv mice and targeted Lrd mutants), left-specific marker genes such as Nodal and Lefty exhibit a complex spectrum of aberrant expression patterns that includes left-sided, right-sided, bilateral, or absent expression in different subsets of embryos (Collignon et al. 1996; Lowe et al. 1996; Meno et al. 1996; Supp et al. 1999). In contrast, mice lacking node cilia or showing aberrant cilia morphology (i.e., targeted Kif3A and Kif3B mutants or mice lacking the ciliary assembly protein Polaris) exhibit bilateral expression or bilateral absence of left-sided genes exclusively, based on the examples reported in the literature (Nonaka et al. 1998; Marszalek et al. 1999; Takeda et al. 1999; Murcia et al. 2000). As we and others have noted previously (Vogan and Tabin 1999; Wagner and Yost 2000; Brueckner 2001), the two classes of mutants, according to the morphogen flow hypothesis, should have the same downstream consequences: the morphogen would be released into a node devoid of flow, whether from a lack of ciliary motility or from an absence of cilia entirely. These differences in molecular phenotypes are therefore not predicted by the morphogen flow hypothesis. They do, however, suggest an alternate view of the early events at the node, as outlined below.

\section{A two-cilia model for L-R axis specification}

Before the discovery of motile monocilia at the mouse node, so-called primary cilia, which are a common feature of polarized epithelial cells, had long been postulated to serve a mechanosensory role. Accordingly, if we hypothesize that the mouse node in fact contains two distinct classes of primary cilia-some which function as flow generators and others that function as mechano- sensors-a simple explanation for the phenotypic differences between the two classes of cilia mutants immediately follows. In particular, mutants such as iv with immotile node cilia would retain functional mechanosensors that could then be triggered in a somewhat random manner by the movement of the embryo, leading to the observed spectrum of molecular phenotypes (Collignon et al. 1996; Lowe et al. 1996; Meno et al. 1996; Supp et al. 1999). In contrast, mutants unable to assemble cilia, such as mice lacking KIF3A, KIF3B, or Polaris (Nonaka et al. 1998; Marszalek et al. 1999; Takeda et al. 1999; Murcia et al. 2000), would lose both the ability to generate flow and the ability to respond to it, leading to a distinct spectrum of phenotypes.

Although it has not received as much attention in the literature, the possibility of a mechanosensory mechanism for receiving and transducing nodal flow has been raised previously, largely as a formal alternative to the morphogen flow hypothesis (Okada et al. 1999; Bruckner 2001). More recent data, however, based on a convergence of studies implicating the polycystic kidney disease gene $P k d 2$ in both early L-R signaling events and in cilia-based mechanoreception in mature kidney cells, allow a compelling case to be made in favor of this alternative model.

Mutations in PKD1 and PKD2 were first identified in humans as the genetic basis for autosomal dominant polycystic kidney disease, a relatively common pathology characterized by the development of multiple renal cysts typically presenting during the third or fourth decade of life (for review, see Igarashi and Somlo 2002). At a molecular level, the PKD1 and PKD2 gene products (termed polycystin-1 and -2) encode multipass transmembrane proteins, and biochemical studies have shown that these proteins physically interact (Qian et al. 1997) and can associate at the plasma membrane to form a calcium-permeable nonselective cation channel (Hanaoka et al. 2000). Additional studies have also identified an intracellular pool of polycystin-2 that localizes to the endoplasmic reticulum, where it has been proposed to function as a calcium-activated calcium release channel (Koulen et al. 2002). A cilia-based role for polycystin-2 is suggested further by the localization of this protein to the primary cilia of both cultured kidney cells and mouse kidney cells in vivo (Pazour et al. 2002), where it colocalizes with other polycystic kidneys disease proteins such as polycystin-1, a novel cytoplasmic protein known as cystin, and the intraflagellar transport protein Polaris (Hou et al. 2002; Yoder et al. 2002). Interestingly, Caenorhabditis elegans homologs of polycystin-1, polycystin-2, and Polaris also colocalize within specialized sensory cilia, where they are required for certain malespecific mating behaviors (Barr and Sternberg 1999; Barr et al. 2001; Haycraft et al. 2001; Qin et al. 2001).

On the basis of these observations and others, it has been proposed that the cilia-based polycystin complex present in kidney cells might serve a mechanosensory function coupled to intracellular calcium signaling (for review, see Igarashi and Somlo 2002). Although conclusive evidence for this hypothesis is lacking, recent stud- 
ies have shown that mechanically deflecting the primary cilium of canine kidney (MDCK) cells in culture results in a strong increase in intracellular calcium (Praetorius and Spring 2001). This response is thought to be initiated by calcium influx through stretch-activated calcium channels and propagated by the release of calcium from intracellular calcium stores (Praetorius and Spring 2001). Although the specific role of the polycystin complex in the initiation and/or propagation of this calcium response remains to be established, these studies, when viewed collectively, indicate that polycystins function in the context of the kidney within primary cilia specialized for mechanosensation.

Significantly, recent reanalysis of the phenotype of mice deficient in $P k d 2$ has also revealed a complex spectrum of laterality defects in these mutants (Pennekamp et al. 2002). In particular, Pkd2 mutant mice fail to activate left-sided genes anteriorly, coupled with a delayed bilateral onset of left-sided genes in the posterior region of the embryo (Pennekamp et al. 2002). This phenotype is strongly reminiscent of those mutants that fail to assemble cilia at the node (Nonaka et al. 1998; Marszalek et al. 1999; Takeda et al. 1999; Murcia et al. 2000), implying that this is the default molecular phenotype that occurs in the absence of cilia-dependent L-R signaling inputs. In light of these results, we propose that a distinct class of mechanosensory cilia, operating in conjunction with polycystins, is required for receiving and transducing the nodal flow signal at the mouse node. According to this "two-cilia" model of L-R axis specification (Fig. 1B), a subset of node cilia are motile and are required to generate nodal flow, whereas a distinct subset of node cilia are required to sense the flow. Because the node is essentially a pit, we anticipate that cilia localized to the left periphery, where the flow impacts the side of the node, would encounter significantly more fluid pressure than those positioned on the right, and that those displaced sufficiently would respond by initiating a calcium-dependent signal transduction event. This signaling event could, in turn, lead to the asymmetric release of one or more key signaling molecules on the left side of the node. Candidates for this asymmetrically released factor include Fgf8, Gdf1, and Nodal (Meyers and Martin 1999; Rankin et al. 2000; Brennan et al. 2002) - the critical L-R determinants previously hypothesized to be secreted symmetrically in the node region and then redistributed asymmetrically in direct response to nodal flow. The fact that Nodal activity is specifically required within node cells to correctly establish the earliest known molecular asymmetries in the node region (Brennan et al. 2002) makes Nodal a particularly attractive candidate for this asymmetrically released node morphogen.

\section{Implications of the two-cilia model}

Although the two-cilia model successfully addresses much of the data inadequately explained by the morphogen flow hypothesis, there are several intriguing findings in the field that remain somewhat enigmatic for both models. Perhaps the most interesting of these relate to the inv mouse, an insertional mutant that exhibits close to a $100 \%$ incidence of L-R reversals (Yokoyama et al. 1993). Paradoxically, the measured flow rate at inv nodes, though decreased relative to wild-type embryos, is not reversed as might be predicted a priori by the nodal flow model (Okada et al. 1999). Consequently, attempts to reconcile these observations with the morphogen flow hypothesis have led to rather ad hoc proposals regarding the biophysical properties of the putative morphogen (Okada et al. 1999). The fact, however, that inv cilia exhibit the same vortical path and speed as wild-type cilia (Okada et al. 1999) indicates that the product of the inv gene, a novel cytoplasmic protein known as inversin (Mochizuki et al. 1998; Morgan et al. 1998), is not required for the motility of node cilia per se, but may instead act downstream of nodal flow in the reception and transduction of the nodal flow signal. In this regard, it is interesting that inv mutants develop a severe kidney pathology reminiscent of other polycystic kidney disease gene mutants (Yokoyama et al. 1993; Mochizuki et al. 1998; Morgan et al. 1998), indicating that these genes function in a common signaling pathway. In addition, although very little is known to date regarding the molecular mechanisms of inversin function, two recent studies have demonstrated a biochemical interaction between inversin and the calcium-binding protein calmodulin (Yasuhiko et al. 2001; Morgan et al. 2002). This interaction occurs in the absence of calcium but is blocked in the presence of elevated calcium levels (Yasuhiko et al. 2001), suggesting that inversin is regulated by calcium-dependent processes that could be linked to the calcium-based output of the mechanosensory cilia proposed herein.

If inversin functions in a calcium-coupled signal transduction event, and the activity of motile primary cilia is not directly affected by this gene product, how does one explain the decreased flow rate and turbulent flow pattern seen in inv mice (Okada et al. 1999)? The explanation may lie in the observation that inv nodes contain aberrant cell masses that appear concentrated to the left and right edges of the node, resulting in a distinct narrowing in the width of the node along the L-R axis (Okada et al. 1999). In principle, these gross alterations in node morphology could result in the observed changes in fluid dynamics, as noted previously /Okada et al. 1999). It is also worth noting that aberrant cell masses are a characteristic feature of the polycystic kidney disease phenotype, indicating that inversin serves an important function in both contexts in the maintenance of normal cellular morphology.

A second set of data that has been difficult to incorporate into the growing framework of the L-R field is the observation that gap junctions have an integral role in the early establishment of L-R asymmetry (Levin and Mercola 1998), acting upstream of the known asymmetrically expressed genes at the chick node (Levin and Mercola 1999). Although there is no obvious way to integrate these observations into the morphogen flow model, there is at least a context for thinking about their 
role within the framework of the two-cilia model. In particular, when a single MDCK cell primary cilium is manipulated in culture, an increase in intracellular calcium is observed not only in the stimulated cell but also in neighboring cells (Praetorius and Spring 2001). Critically, this secondary wave of calcium release has been shown to depend on gap junction activity (Praetorius and Spring 2001). Therefore, the published observations on the role of gap junctions in L-R asymmetry could reflect a requirement for gap junctions in the vicinity of the node for the propagation and enhancement of the initial mechanosensory signal. Moreover, the lack of expression of the gap junction protein Connexin-43 within the node itself (Levin and Mercola 1999) could serve as a barrier to the spread of calcium activation from the left to the right side of the node following mechanosensory stimulation.

It is also interesting to consider the two-cilia model in the context of recent studies identifying differences in membrane potentials between the left and right halves of the node and primitive streak in chick (Levin et al. 2002). Interestingly, this asymmetric membrane depolarization coincides both temporally and spatially with the appearance of Lrd transcripts and monocilia in chick (Essner et al. 2002). Accordingly, we suggest that these asymmetric membrane depolarization events and the observed sensitivity of chick embryos to $\mathrm{H}^{+} / \mathrm{K}^{+}$-ATPase channel blockers (Levin et al. 2002) may be linked to the calciumcoupled cilia-based signaling events proposed herein. There is no obvious way, on the other hand, to reconcile either of the cilia-based models with the asymmetry in $\mathrm{H}^{+} / \mathrm{K}^{+}$-ATPase $\alpha$ subunit mRNA localization reportedly present in Xenopus embryos at the 4-cell stage (Levin et al. 2002). If the latter observation is correct, it would argue that a mechanism unlinked to cilia operates upstream of the earliest asymmetric gene expression patterns in Xenopus. It is worth noting, however, that both cilia and $L r d$ transcripts have been localized to organizer derivatives in both frog and fish embryos (Essner et al. 2002), arguing that at least some aspects of node cilia function might be common to all vertebrates.

In summary, much of the appeal inherent to the nodal flow model lies in its ability to explain, in principle, how handed asymmetry might arise from a ground state condition of bilateral asymmetry or antisymmetry (for review, see Vogan and Tabin 1999|. Based mostly on work in mice, a general picture is now emerging of how this might take place-however, the actual mechanistic details underlying this emergence of handed asymmetry, as manifested by the appearance of nodal flow, remain obscure at best. It will also be important to determine whether the coupled processes of nodal flow and mechanoreception represent a universal mechanism for specifying the vertebrate L-R axis or whether this system is specific to mammals. Whereas recent studies hint at the apparent conservation of several key components of this system (Yasuhiko et al. 2001; Essner et al. 2002; Morgan et al. 2002), further work is clearly needed to resolve this important issue. In the meantime, the consensus view emerging from the mouse studies outlined here points at a critical role for node cilia both in the generation and the reception of nodal flow signals, providing an elegant and compelling model for vertebrate L-R axis specification.

\section{Acknowledgments}

We thank Joe Yost, Hiroshi Hamada, Chris Wright, Liz Robertson, and especially Martina Brueckner for many helpful discussions on this topic. Work in C.J.T.'s laboratory on L-R asymmetry is supported by a grant from the National Institutes of Health (HL56485).

\section{References}

Afzelius, B.A. 1976. A human syndrome caused by immotile cilia. Science 193: 317-319.

Barr, M.M. and Sternberg, P.W. 1999. A polycystic kidney-disease gene homologue required for male mating behaviour in C. elegans. Nature 401: 386-389.

Barr, M.M., DeModena, J., Braun, D., Nguyen, C.Q., Hall, D.H., and Sternberg, P.W. 2001. The Caenorhabditis elegans autosomal dominant polycystic kidney disease gene homologs lov-1 and $p k d-2$ act in the same pathway. Curr. Biol. 11: 1341-1346.

Bartoloni, L., Bloiun, J.L., Pan, Y., Gehrig, C., Maiti, A.K., Scamuffa, N., Rossier, C., Jorissen, M., Armengot, M., Meeks, M., et al. 2002. Mutations in the DNAH11 (axonemal heavy chain dynein type 11) gene cause one form of situs inversus totalis and most likely primary ciliary dyskinesia. Proc. Natl. Acad. Sci. 99: 10282-10286.

Bellomo, D., Lander, A., Harragan, I., and Brown, N.A. 1996. Cell proliferation in mammalian gastrulation: The ventral node and notochord are relatively quiescent. Dev. Dyn. 205: 471-485.

Brennan, J., Norris, D.P., and Robertson, E.J. 2002. Nodal activity in the node governs left-right asymmetry. Genes \& Dev. 16: $2339-2344$.

Brown, N.A. and Wolpert, L. 1990. The development of handedness in left/right asymmetry. Development 109: 1-9.

Brueckner, M. 2001. Cilia propel the embryo in the right direction. Am. J. Med. Genet. 101: 339-344.

Burdine, R.D. and Schier, A.F. 2000. Conserved and divergent mechanisms in left-right axis formation. Genes \& Dev. 14: $763-776$.

Capdevila, J., Vogan, K.J., Tabin, C.J., and Izpisúa Belmonte, J.C. 2000. Mechanisms of left-right determination in vertebrates. Cell 101: 9-21.

Collignon, J., Varlet, I., and Robertson, E.J. 1996. Relationship between asymmetric nodal expression and the direction of embryonic turning. Nature 381: 155-158.

Essner, J.J., Vogan, K.J., Wagner, M.K., Tabin, C.J., Yost, H.J., and Brueckner, M. 2002. Conserved function for embryonic nodal cilia. Nature 418: 37-38.

Guichard, C., Harricane, M.C., Lafitte, J.J., Godard, P., Zaegel, M., Tack, V., Lalau, G., and Bouvagnet, P. 2001. Axonemal dynein intermediate-chain gene (DNAI1) mutations result in situs inversus and primary ciliary dyskinesia (Kartegener syndrome). Am. J. Hum. Genet. 68: 1030-1035.

Hanaoka, K., Qian, F., Boletta, A., Bhunia, A.K., Piontek, K., Tsiokas, L., Sukhatme, V.P., Guggino, W.B., and Germino, G.G. 2000. Co-assembly of polycystin-1 and -2 produced unique cation-permeable currents. Nature 408: 990-994.

Haycraft, C.J., Swoboda, P., Taulman, P.D., Thomas, J.H., and Yoder, B.K. 2001. The C. elegans homolog of the murine cystic kidney disease gene $\operatorname{Tg} 737$ functions in a ciliogenic pathway and is disrupted in osm-5 mutant worms. Development 128: 1493-1505. 
Hou, X., Mrug, M., Yoder, B.K., Lefkowitz, E.J., Kremmidiotis, G., D'Eustachio, P., Beier, D.R., and Guay-Woodford, L.M. 2002. Cystin, a novel cilia-associated protein, is disrupted in the cpk mouse model of polycystic kidney disease. J. Clin. Invest. 109: 553-540.

Igarashi, P. and Somlo, S. 2002. Genetics and pathogenesis of polycystic kidney disease. J. Am. Soc. Nephrol. 13: 2384-2398.

Koulen, P., Cai, Y., Geng, L., Maeda, Y., Nishimura, S., Witzgall, R., Ehrlich, B.E., and Somlo, S. 2002. Polycystin-2 is an intracellular calcium release channel. Nat. Cell Biol. 4: 191-197.

Levin, M. and Mercola, M. 1998. Gap junctions are involved in the early generation of left-right asymmetry. Dev. Biol. 203: $90-105$.

1999. Gap junction-mediated transfer of left-right patterning signals in the early chick blastoderm is upstream of Shh asymmetry in the node. Development 126: 4703-4714.

Levin, M., Johnson, R.L., Stern, C.D., Kuehn, M., and Tabin, C. 1995. A molecular pathway determining left-right asymmetry in chick embryogenesis. Cell 82: 803-814.

Levin, M., Thorlin, T., Robinson, K.R., Nogi, T., and Mercola, M. 2002. Asymmetries in $\mathrm{H}^{+} / \mathrm{K}^{+}$-ATPase and cell membrane potentials comprise a very early step in left-right patterning. Cell 111: 77-89.

Lowe, L.A., Supp, D.M., Sampath, K., Yokoyama, T., Wright, C.V., Potter, S.S., Overbeek, P., and Kuehn, M.R. 1996. Conserved left-right asymmetry of nodal expression and alterations in murine situs inversus. Nature 381: 158-161.

Marszalek, J.R., Ruiz-Lozano, P., Roberts, E., Chien, K.R., and Goldstein, L.S. 1999. Situs inversus and embryonic ciliary morphogenesis defects in mouse mutants lacking the KIF3A subunit of kinesin-II. Proc. Natl. Acad. Sci. 96: 5043-5048.

Meno, C., Saijoh, Y., Fujii, H., Ikeda, M., Yokoyama, T., Yokoyama, M., Toyoda, Y., and Hamada, H. 1996. Left-right asymmetric gene expression of the TGF $\beta$-family member lefty in mouse embryos. Nature 381: 151-155.

Meyers, E.N. and Martin, G.R. 1999. Differences in left-right axis pathways in mouse and chick: Functions of FGF8 and SHH. Science 285: 403-406.

Mochizuki, T., Saijoh, Y., Tsuchiya, K., Shirayoshi, Y., Takai, S., Taya, C., Yonekawa, H., Yamada, K., Nihei, H., Nakatsuji, N., et al. 1998. Cloning of inv, a gene that controls left/right asymmetry and kidney development. Nature 395: $177-181$

Morgan, D., Turnpenny, L., Goodship, J., Dai, W., Majumder, K., Matthews, L., Gardner, A., Schuster, G., Vien, L., Harrison, W., et al. 1998. Inversin, a novel gene in the vertebrate left-right axis pathway, is partially deleted in the inv mouse. Nat. Genet. 20: 149-156.

Morgan, D., Goodship, J., Essner, J.J., Vogan, K.J., Turnpenny, L., Yost, H.J., Tabin, C.J., and Strachan, T. 2002. The left-right determinant inversin has highly conserved ankyrin repeat and IQ domains and interacts with calmodulin. Hum. Genet. 110: $377-384$.

Murcia, N.S., Richards, W.G., Yoder, B.K., Mucenski, M.L., Dunlap, J.R., and Woychick, R.P. 2000. The Oak Ridge Polycystic Kidney (orpk) disease gene is required for left-right axis determination. Development 127: 2347-2355.

Nonaka, S., Tanaka, Y., Okada, Y., Takeda, S., Harada, A., Kanai, Y., Kido, M.., and Hirokawa, N. 1998. Randomization of left-right asymmetry due to loss of nodal cilia generating leftward flow of extraembryonic fluid in mice lacking KIF3B motor protein. Cell 95: 829-837.

Nonaka, S., Shiratori, H., Saijoh, Y., and Hamada, H. 2002. Determination of left-right patterning of the mouse embryo by artificial nodal flow. Nature 418: 96-99.

Olbrich, H., Haffner, K., Kispert, A., Volkel, A., Volz, A.,
Sasmaz, G., Reinhardt, R., Hennig, S., Lehrach, H., Konietzko, N., et al. 2002. Mutations in DNAH5 cause primary ciliary dyskinesia and randomization of left-right asymmetry. Nat. Genet. 30: 143-144.

Okada, Y., Nonaka, S., Tanaka, Y., Saijoh, Y., Hamada, H., and Hirokawa, N. 1999. Abnormal nodal flow precedes situs inversus in iv and inv mice. Mol. Cell 4: 459-468.

Pazour, G.J., San Agustin, J.T., Follit, J.A., Rosenbaum, J.L., and Witman, G.B. 2002. Polycystin-2 localizes to kidney cilia and the ciliary level is elevated in orpk mice with polycystic kidney disease. Curr. Biol. 12: R378-R380.

Pennarun, G., Escudier, E., Chapelin, C., Bridoux, A.M., Cacheux, V., Roger, G., Clement, A., Goossens, M., Amselem, S., and Duriez, B. 1999. Loss-of-function mutations in a human gene related to Chlamydomonas reinhardti dynein IC78 result in primary ciliary dyskinesia. Am. I. Hum. Genet. 65: 1508-1519.

Pennekamp, P., Karcher, C., Fischer, A., Schweickert, A., Skryabin, B., Horst, J., Blum, M., and Dworniczak, B. 2002. The ion channel polycystin-2 is required for left-right axis determination in mice. Curr. Biol. 12: 938-943.

Praetorius, H.A. and Spring, K.R. 2001. Bending the MDCK primary cilium increases intracellular calcium. J. Membrane Biol. 184: 71-79.

Qian, F., Germino, F.J., Cai, Y., Zhang, X., Somlo, S., and Germino, G.G. 1997. PKD1 interacts with PKD2 through a probable coiled-coil domain. Nat. Genet. 16: 179-183.

Qin, H., Rosenbaum, J.L., and Barr, M.M. 2001. An autosomal recessive polycystic kidney disease gene homolog is in volved in intraflagellar transport in C. elegans ciliated sensory neurons. Curr. Biol. 11: 457-461.

Rankin, C.T., Bunton, T., Lawler, A.M., and Lee, S.-J. 2000. Regulation of left-right patterning in mice by growth/differentiation factor-1. Nat. Genet. 24: 262-265.

Sulik, K., Dehart, D.B., Inagaki, T., Carson, J.L., Vrablic, T., Gesteland, K., and Schoenwolf, G.C. 1994. Morphogenesis of the murine node and notochordal plate. Dev. Dyn. 201: 260-278.

Supp, D.M., Witte, D.P., Potter, S.S., and Brueckner, M. 1997. Mutation of an axonemal dynein affects left-right asymmetry in inversus viscerum mice. Nature 389: 963-966.

Supp, D.M., Brueckner, M., Kuehn, M.R., Witte, D.P., Lowe, L.A., McGrath, J., Corrales, J., and Potter, S.S. 1999. Targeted deletion of the ATP binding domain of left-right dynein confirms its role in specifying development of left-right asymmetries. Development 126: 5495-5504.

Takeda, S., Yonekawa, Y., Tanaka, Y., Okada, Y., Nonaka, S., and Hirokawa, N. 1999. Left-right asymmetry and kinesin superfamily protein KIF3A: New insights in determination of laterality and mesoderm induction by $k i f 3 A^{-/-}$mice analysis. J. Cell Biol. 145: 825-836.

Vogan, K.J. and Tabin, C.J. 1999. A new spin on handed asymmetry. Nature 397: 295-298.

Wagner, M.K. and Yost, H.J. 2000. Left-right development: The roles of nodal cilia. Curr. Biol. 10: R149-R151.

Yasuhiko, Y., Imai, F., Ookubo, K., Takakuwa, Y., Shiokawa, K. and Yokoyama, T. 2001. Calmodulin binds to inv protein Implication for the regulation of inv function. Develop. Growth Differ. 43: 671-681.

Yoder, B.K., Hou, X., and Guay-Woodford, L.M. 2002. The polycystic kidney disease proteins, polycystin-1, polycystin-2, polaris, and cystin, are co-localized in renal cilia. J. Am. Soc. Nephrol. 13: 2508-2516.

Yokoyama, T., Copeland, N.G., Jenkins, N.A., Montgomery, C.A., Elder, F.F.B., and Overbeek, P.A. 1993. Reversal of leftright asymmetry: A situs inversus mutation. Science 260: 679-682. 


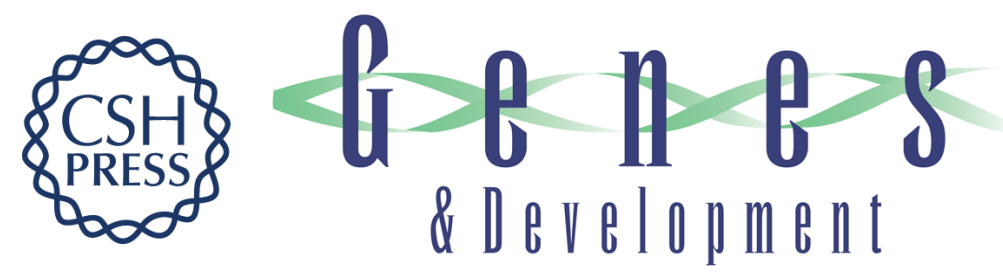

\section{A two-cilia model for vertebrate left-right axis specification}

Clifford J. Tabin and Kyle J. Vogan

Genes Dev. 2003, 17:

Access the most recent version at doi:10.1101/gad.1053803

References This article cites 50 articles, 15 of which can be accessed free at: http://genesdev.cshlp.org/content/17/1/1.full.html\#ref-list-1

License

Email Alerting Receive free email alerts when new articles cite this article - sign up in the box at the top Service right corner of the article or click here.

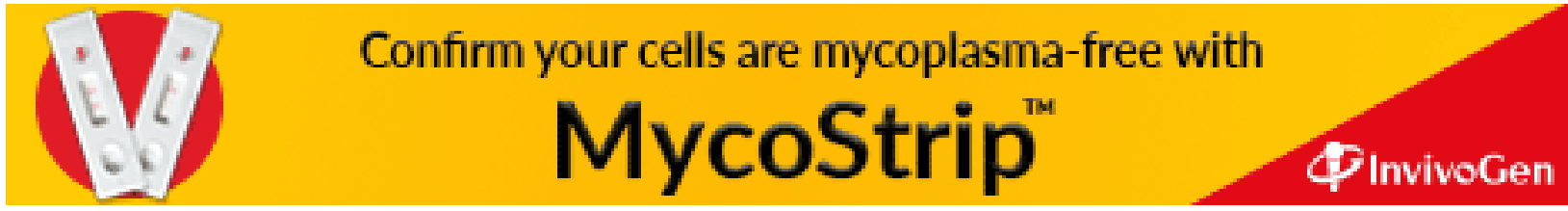

